\title{
XXXII. Mittheilungen aus dem mineralogischen Institut der Universität Strassburg. 14.
}

\author{
Ueber Baryt aus dem Kronthal im Elsass; natïrliche und künstliche \\ Aetzflguren.
}

Von

\author{
J. Valentin in Strassburg
}

(Mit 8 Figuren im Text.)

Im Kronthal bei Wasselnheim im Elsass finden sich auf einzelnen Spalten im mittleren Buntsandstein, welcher dort in grossen Bruchen ausgebeutet wird, neben grossen, weissen, rauhfächigen Schwerspathkrystallen, die schon länger von dort bekannt sind, auch sehr schöne dul'chsichlige Krystalle, die meines Wissens noch nirgends Erwähnung gefunden haben. Die Krystalle sind ungefäbr $\frac{1}{4}$ bis $1 \mathrm{~mm}$ dick, 1 bis $20 \mathrm{~mm}$ lang und $\frac{3}{4}$ bis $15 \mathrm{~mm}$ breit. Sie sind wasserhell his blassgrün durchsichtig, sämmtlich nach der Basis dünntafelförmig entwickelt, und von der Seite her so aufgewachsen, dass sie in der Regel nur an dem einen Ende der $a$ - und $b$-Axe

Fig. 1.

frei ausgebildet sind. Die gewöhnliche Combination (Fig. 1) ist $c=\{001\} 0 P, m=\{110\} \infty P$ und $d=$ $\{102\} \frac{1}{2} \bar{P} \infty$, wozu in vielen Fällen noch $o=\{011\} \breve{P}_{\infty}$, seltener $b=\{010\} \infty \breve{P}_{\infty}$ trill. Formen mil complicirten Zeichen kommen an vereinzelten Krystallen meist nur mit einer Fläche ausgebildet vor. Die Basis ist besonders an den grösseren Krystallen ganz von Vicinalflächen bedeckt, von denen einige aus der Zone (Basis, Makropinakoid) auch messbar waren; sie giebt daher gewöhnlich mehrere Reflexe.

Das Prisma ist gut ausgebildet, zuweilen matt durch zarte Streifen, welche der Kante $\{110\}\{001\}$ parallel laufen. Je nur mil einer Fläche entwickelt sind an den untersuchlen Krystallen die Prismen $z=\{130\} \infty \breve{P} 3$ 
und $\eta=\{320\} \infty \bar{P} \frac{3}{2} ; \approx$ und $\eta$ sind stark glänzend. Das Brachypinakoid $b=\{010\} \infty \breve{P}_{\infty}$ wurde nur selten als sebr kleine, glänzende Fläche beobachtet. Das Makrodoma $d=\{102\}_{\frac{1}{2}} \vec{P} \infty$ und das Brachydoma $o=\{011\} \breve{P}_{\infty}$ Ireten ganz regelmässig mit kleinen, gut spiegelnden Flächeu auf: Auch die Grundpyramide $z=\{111\} P$ erscheint vollfächig ausgebildet, als $\mathrm{Ab}-$ slumpfung der Kanten $\{001\},\{110\}$.

Die an den gemessenen Krystallen bestimmten und berechneten Winkel sind folgende :

\begin{tabular}{|c|c|c|}
\hline & Gemessen : & Berechnet \\
\hline$m: m=(110)(1 T 0)=$ & $={ }^{*} 78^{0} \mathrm{Z}^{\prime}$ & - \\
\hline$o: o=(011) \cdot\left(0 T^{\top} 1\right)=$ & $=* 10523$ & - \\
\hline$m: \eta=(110):(320)=$ & $=1035$ & $10^{\circ} 40^{\prime}$ \\
\hline$m: z=(110):(130)$ & 2844 & 2835 \\
\hline$d: d=(102):(10 \overline{2})=$ & $=10215$ & 10218 \\
\hline$z: c=(111):(001)=$ & 6428 & 64. 18 \\
\hline$z: o=(111):(011)=$ & 4430 & $44 \quad 19$ \\
\hline
\end{tabular}

Zur Berechnung des Axenverhältnisses wurden die Winkel des Brachydomas $o$ und des Prismas $m$ benutzt, da die Winkelwerthe zwischen den genannten Flächen an zweien der gemessenen Krystalle auf die Minute ubereinstimmen. Es ergab sich :

$$
a: b: c=0,8146: 1: 1,3119 .
$$

Der wabre Winkel der optischen Axen wurde fur $\mathrm{Na}$-Licht $2 V_{a}=$ $40^{\circ} 58^{\prime}$ bestimmt, aus dem spitzen und stumpfen in Alphamonobromnaphtalin gemessenen scheinbaren Axenwinkel $H_{a}=20^{\circ} 25^{\prime}$ und $H_{0}=69^{\circ} \mathrm{y}^{\prime}$.

Der miltlere Brechungsquotient berechnet sich aus diesen Daten und dem Brechungsquotienten des Alphamonobromnaphtalins $n=1,654$ als $\beta=1,649$.

Der von dem gewöhnlichen stark abweichende wabre Axenwinkel bestimmte mich, eine Prüfung auf Strontium vorzunehmen. Es konnte indess ein Gehalt an diesem Elemente spectralanalytisch nicht nacbgewiesen werden und lässt sich dẹmnach über die Ursache der auch an anderen Barytvorkommnissen vielfach beobachteten Abweichung des optischen Axenwinkels von dem gewöhnlich angegebenen nichts Positives behaupten.

Eine etwas eingehendere Betrachtung erheischen die an den oben beschriebenen Krystallen auftrelenden Vicinalflächen und Aetzerscheinungen. An einem Krystalle wurde auf der Basis $\psi=\{1.0 .20\}_{\frac{1}{20}} \vec{P} \infty^{*}$; , an einem anderen $\psi^{1}=\{1.0 .50\}_{\frac{1}{0}} \bar{P}_{\infty} \infty$ bestimmt. Letztere Form trilt nur mit den

*) Vergl. für diese und alle folgenden noch zu besprechenden Flächen die nachstehende Winkellabelle:

Grotb, Zeitschrift f. Krystallogr. $x Y$. 
beiden Flächen (1.0.50) und (T.0.5̄0 ) auf. Zugleich zeigt letzterer Krystall auf der Basis und dem genannten Doma eine Streifung, welche parallel der Prismenkante und der Makrodiagonale verläuft und bei Fig. 2.

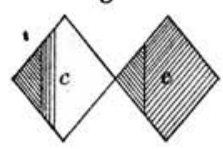
dem in Fig. 2 angedeuteten Auftreten jede Symmetrie aufhebt. - Auch besitzen eine grosse Anzahl von Krystallen noch einige im Folgenden näher zu besprechende matle, oft gerundete oder windschiefe Flächen, von denen nur wenige eben genug waren, um gemessen zu werden. Zu diesen Flächen gehört in der Prismenzone das Prisma $m^{3}=$ $\{750\}$, welches dem als glänzende Fläche ausgehildelen $\{310\}$ ziemlich nahe kommt, sowie ein nur mit einer Fläche entwickeltes Prisma $m^{2}=\{36.35 .0\}$.

Ferner tritt als Aetzläche $z\{111\}$ auf; sie unterscheidet sich von der vorher erwähnten ursprünglichen Form $z\{111\}$ durch eine matte, raube Oberflächenbeschaffenheit und grössere Entwicklung ihrer Flächen. In der Zone Basis Makropinakoid ein Makrodoma $u^{1}=\{905\}{ }_{5}^{\circ} \bar{P} \infty$. Am häufigsten treten diese matten Flächen an der Kante $\{001\}\{110\}$ auf.

Die Art der Ausbildung der eben genannten Formen, insbesondere die Rauhigkeil ihrer Oberfläche und die häufig concave Beschaffenheit derselben veranlasslen mich, sie als natürliche Aetzflächen anzusprechen. Es kommt noch hinzu, dass sie sich besonders an solchen Krystallen finden, auf denen eine andere Erscheinung beobachtet wurde, welche, wie gezeigt werden soll, auf naturliche Aetzfiguren zurückzufuhren ist.

Auf der Basis treten bei theilweise abgeblendetem

Fig. 3.

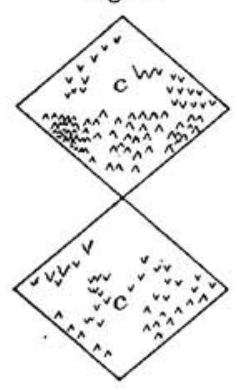
Lichte unter dem Mikroskop eigenthumliche Zeichnungen deutlich hervor (Fig. 3). Erst bei schwacher Vergrösserung zeigen sich gewisse Partien der Basis von parallelen Zickzacklinien bedeckt, bei stärkerer Vergroösserung lösen diese sich in einzelne dachförmige bis spitzbogenartige, äusserst zarle, monosymmetrische Figuren auf. Die Anordnung auf der Basis wechselt; constant scheint nur, dass die Halbirungslinie des Winkels an der Spitze der $a$-Axe parallel läuft. Zuweilen ist nahezu die ganze Basisläche mit den Figuren uberzogen, deren Spitze nach einem Ende der $a$-Axe weist, und Figuren, welche eine umgekehrte Lage besitzen, sind auf einen ganz kleinen Raum beschränkt; zuweilen beobachtel man auch einen drei- bis vierfachen Wechsel in der Orientirung der Spitzen. Die Grenze zwischen den entgegengesetzt orientirten Figuren verläuft immer geradlinig. Entweder berăhren sich die mit Figuren bedeckten Theile der Fläche direct, oder sie sind durch glatle Zonen getrennt. Ueberhaupt ist die Bedeckung mit den Figuren eine sehr ungleichmässige; stellenweise sind sie dichtgedrängt und dann gelegentlich auch wieder ganz vereinzelt auf derselben Krystallfäche. Manche 
Krystalle erscheinen bei oberflächlicher Betrachlung ganz frei von den Figuren, erst bei intensivem Suchen entdeckt man sie schwach angedeutet in irgend einer Ecke der Basis.

Dabei verhalten sich die beiden Basislächen oft verschieden. Krystalle, an welchen nur die eine Orientirung auf einer Fläche herrschte, wurden nicht beobachtet.

Der Winkel an der Spitze der Figuren schwankt zwischen $50^{\circ}$ und $60^{\circ}$. Durch Verschiebung des Tubus konnte festgestellt werden, dass die Figuren durch Vertiefungen und nicht etwa durch Erbabenheiten gebildet werden.

Die Figuren finden sich, wie schon erwähnt, sehr häufig mit den malten windscbiefen Flächen zusammen, sind aber stets auf die Basis beschränkt.

Beziehungen zu kúnstlichen, sowie Vergleiche mit naturlichen Aetzfiguren an Barytkrystallen von andern Fundorten berechtigen wohl zu der Annabme, dass es sich hei diesen Erscheinungen um natürliche Aetzfiguren handelt. So bildet Bu s z*) auf dem Brachydoma $o=\{011\}$ eines Baryts von Mittelagger auftretende Figuren ab, welche in ihrer Erscheinungsweise den Kronthaler Basisfiguren ähnlich sind, die er aber für Wachsthumserscheinungen hält.

Ferner zeigen einige Barylkrystalle, welche sich in der Sammlung des hiesigen mineralogischen Instituts befinden, ganz ausgezeichnete makroskopische Aetzfiguren, welche etwas eingehender besprochen werden mögen.

In zwei Stufen vertreten ist ein Vorkommen von Pallaflat bei St. Bess in Cornwall. Die bläulichgrünen Krystalle sind prismatisch nach der a-Axe gestreckt und erreicben eine Länge von $20 \mathrm{~cm}$. Sie zeigen die Flä́chen des Prismas $m=\{110\} \infty P$, des Brachydomas $o=\{011\} \infty \breve{P}$ und der Basis $c=\{001\} 0 P$. Sämmlliche Flächen siud so stark corrodirt, dass die mit dem Anlegegoniometer gemessenen Winkel an den verschiedenen Stellen um $5-10^{\circ}$ schwankten. Die Basis scheint nur wenig angegriffen, während die Prismen vollständig mit tiefen aber nicht regelmässig begrenzten Grubchen bedeckt sind:- Auffallend sind die Aetzfiguren auf den Flächen $\{011\}$. Soweit hier die Corrosion uberhaupt bestimmle Formen erzeugt hat, erkennt man dreieckige Vertiefungen (Fig. 4), deren Anordnung der. Symmetrie des rhombischen Systems entspricht. Diese Vertiefungen werden gebildet im Grunde von einer dreieckigen Fläche, welche, da sie stets zugleich mit $\{011\}$ spiegelt, als dieser Form zugehörig angeseben wurde, an den Seilen von Makropyramiden, die das Zeichen $Y=\{22.20 .55\}$ erhielten.

Zur Messung der Aetzflächen wurden Ausgüsse der Hoblformen mil

*) Dicse Zeilschr. 10, 37. 
Wood'schem Metall gemacht, und an diesen Ausgussen der Winkel von $o\{011\}$ zu $\{22.20 .55\}$ und der ebene Winkel auf $\{011\}$, gebildet durch die

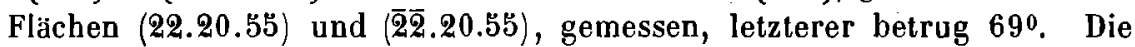
Aetzfiguren sind zum Theil tief in den Krystall eingefressen, zum Theil uberziehen sie dessen Oberfläche wie ein äusserst zarter Hauch. In diesem Falle sind sie den oben erwähnten Basisfiguren des Baryls vom Kronthal durchaus ähnlich; nur finden jene sich auf dem Brachydoma. Nalurliche Aetzfiguren zeigen auch einige Krystalle von Teplitz, die im Hahitus vollständig mit den von Becke*) beschriebenen Baryten aus den Teplitzer Thermalquellen ubereinstimmen. Nur an einer der Stufen sitzt nocb Gesteinsmasse, anscbeinend Porphyr, an; bezuglich der andern bleibt es also unentschieden, ob sie, wie La ub $e^{* *}$ ) von Teplitzer Baryten angiebt, auf cenomanem Hornsteinconglomerat aufgesessen baben, oder auch dem Porphyr entstammen. Die Farbe ist dunkel honiggelb, der grösste der Krystalle ist circa $4 \mathrm{~cm}$ lang und breit und 1,2 cm dick. Alle sind nach der Basis dicktafelförmig. Beobachtete Formen sind $c=\{001\} 0 P, m=\{110\} \infty P$, $o=\{011\} \breve{P}_{\infty}, d=\{102\} \frac{1}{2} \bar{P} \infty, u=\{101\} \bar{P} \infty$ und $a=\{100\} \infty \bar{P} \infty$. $\{110\}$ ist zwar matt, wie Becke von seinen Stucken angiebt, aber die Corrosionsgrübchen zeigen nicht die scharfe Umgrenzung. Die Formen $\{102\}$ und $\{011\}$ sind gross ausgebildet und glänzend, $\{101\}$, welches an den Becke'schen Krystallen fehlt, ist etwas gerundet. Auf $\{011\}$ sitzen mil viereckiger breiter Basis flache Pyramiden auf, mil einer Basiskante der Kante $\{001\}\{011\}$ parallel, wie sie in ähnlicher Weise von Gon nard***) auf $\{102\}$ erwähnt werden. Als Zuschärfung der beiden spitzen Ecken

Fig. 5.

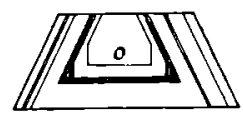
tritt eine Fläche auf (Fig. 5), welche nach ihrer Orientirung von $\{111\}$ oder einer Brachypyramide $\breve{\boldsymbol{P}} m\{l c h h\}$ gebildet sein könnte. Zwei der mir vorliegenden Teplitzer Krystalle lassen einen ausgezeichneten Schalenaufbau erkennen. Der durchsichtige Kern zeigt da, wo die trübe äussere Hülle abgesprengt ist, die glänzenden Krystallflächen des Prismas und des Brachypinakoids.

Die schärfsten Aetzeindrucke zeigt eine Barytstufe vom Giftberge bei Ilorsowitz. Die Krystalle von wasserheller bis bläulicher Farbe sind dicktafelartig nach der Basis und besitzen die Combination $c=\{001\} 0 P, m=$ $\{110\} \infty P, o=\{011\} \breve{P}_{\infty}$ und $\frac{1}{2} d\{102\} \bar{P} \infty$. Die Basis trägt bis zu $2 \mathrm{~mm}$ lange und $1 \mathrm{~mm}$ breite, rhombische Grubchen (s. Fig. 6), deren Längsaxe der b-Axe parallel läuft, und deren Begrenzung durch vier Flächen

*) Becke, Barylkryslalle i. d. Quellengeb. d. Tepl. Thermen. Tscherm. min. u. petr. Mitlh. 1883, 5, 82. Ref. in dieser Zeitschr. 9, 221.

**) La u b e, Verh. d. k. k. geol. Reichsanst. Wien 1883, 85 ,

***) Gon nard, Bull. de la soc. fr. de Min. 1888, 11, 274 . 
einer Pyramide der verticalen Reihe gebildet wird. Die Flächen waren so eben, dass eine Messung mit dem Reflexionsgoniometer möglich war und zwar wurden auch hier die Ausgüsse der Hohlformen mit Wood'schem Metall gemessen. Die Pyramide hat das Zeichen $\Omega=\{$ 2.2.21\}:

Das Prisma ist parallel der Combinationskante mit $\{001\}$ gerieft und zeigt an manchen Stellen aufgewachsene flache Pyramiden von rechteckiger Basis; die eine Basiskante verläuft parallel der c-Axe. Auf dem Makrodoma $\{102\}$ 'sind weniger scharfe dreieckige Verliefungen (s. Fig. 6 oben) vorhanden, deren Spilzen nach der Kante $\{001\}$ \{102\} gerichtel sind; sie entsprechen den von Gon a r d an Barytkrystallen des Puy-de-Dóme auf

Fig. 6.

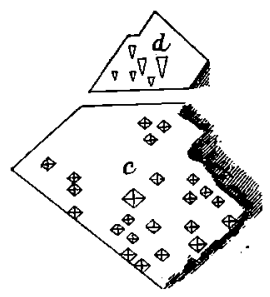
\{102\} beobachteten Figuren in Gestalt und Orientirung.

Soweit mir die Literatur daruber bekannt und zugánglich ist, sind naturliche Aetzfiguren am Baryt bis jetzt von Gonnard und Becke auf $\{110\},\{102\}$ und $\{011\}$ beschrieben. Es sind nunmehr auch noch natürliche Aetzfiguren auf $\{001\}$ beobachtet, und zwar einmal die dachförmigen des Kronthaler Vorkommens und zweitens die rhombenförmigen des Giftberger Baryts. Krystallographisch bestimmt war von Aetzflächen allein das Makropinakoid $a\{100\}$, in welchem Becke an den Teplitzer Krystallen eine Aetzfläche annehmen zu können glauble. Als durch Aetzung hervorgerufen sind auch die oben besprochenen Formen anzusehen, nämlich :

$$
\begin{gathered}
m^{2}=\{36.35 .0\} \infty \bar{P}_{36}^{36}, m^{3}=\{750\} \infty \bar{P}_{\frac{7}{5}}, o=\{011\} \breve{P}_{\infty}, u^{1}=\{905\} \frac{9}{5} \bar{P} \infty \\
z=\{111\} P, \Omega=\{2.21 .21\} \frac{2}{21} P, x=\{22.20 .55\} \frac{2}{5} \bar{P} \frac{11}{10} .
\end{gathered}
$$

\begin{tabular}{|c|c|c|c|}
\hline & & Gemessen : & Berechnet \\
\hline$m: m^{2}=$ & $(110):(36 . \overline{3} \overline{5} .0)$ & $=77^{0} 38^{\prime}$ & $77^{0} 33^{\prime}$ \\
\hline$m: m^{3}=$ & $(110):(750)$ & $=99$ & 858 \\
\hline$z: c=$ & $(111):(001)$ & $=6349$ & $64 \quad 18$ \\
\hline$d: u^{1}=$ & $(102):(90 \overline{5})$ & $=70 \quad 10$ & 70 \\
\hline \multicolumn{4}{|c|}{ 1I. Vicinaltlächen. } \\
\hline$c: \Psi=$ & $(001):(1.0 .20)$ & $=4047^{\prime}$ & $4036^{\prime}$ \\
\hline$c: \Psi^{1}=$ & $(001):(1.0 .50)$ & $=152$ & 151 \\
\hline
\end{tabular}

I. Aetzflächen.

Aetzflächen am Baryt vom Giftberge bei Horsowitz*).

$$
\begin{aligned}
& \Omega: \Omega=(2.2 .21):(\overline{2} .2 .21)=17053^{\prime} \quad 17020^{\prime} \\
& \Omega: \Omega=(2.2 .21):(2 . \overline{2} .21)=14 \quad 7 \quad 14 \quad 4
\end{aligned}
$$

*) Bei der Berechnung wurde das Axenverhältniss $a: b: c=0,8146: 1: 1,3119$ zu Grunde gelegt. 


$$
\begin{aligned}
& \text { Aetzflàchen am Baryt von Cornwall (Pallaflat). } \\
& r: o=(22.20 .55):(011)=39^{\circ} 46^{\prime} \text { Gem. 39022' Ber. }
\end{aligned}
$$

An dem Kronthaler Vorkommen, von dem mir reichliches Material zur Verfugung stand, wurden auch kunstliche Aetzversuche gemacht. Sie wurden ausgefubrt mit kohledsaurem Alkali, ferner mit Aetznatron und mit concentrirter Schwefelsăure.

Das kohlensaure Alkali erwies sich als das schwächste Reagens. Die Krystalle wurden von 3 Minuten bis zu einer Stunde in einer concentrirten Lösung von kohlensaurem Kali oder Natron gekocht. Es gelang nicht auf der Basis die Figuren zu erzielen, welche Ts chermak*) auf ihr angiebt; aber es hatte den Anschein, als ob die Zahl der oben erwähnten dachförmigen Eindrucke (Fig. 3, S: 578) durch die Aetzung vermehrt wurde, und zwar dana, wenn der Krystall nur kurze Zeit, etwa 3 Minuten, mit dem Aetzmittel behandelt war; bei längerer Einwirkung war die Basis stark, aber unregelmässig corrodirt.

Die Prismen waren nach längerer Aetzung mit kleinen Grübchen bedeckt, welche mit den von Tschermak abgebildeten ubereinstimmen. Das Makrodoma $\{102\}$ zeigt Figuren, welche in Form und Anordnung den natürlichen auf dieser Flache gleichkommen.

Die besten Erfolge wurden mit Aetznatron erzielt. Die Krystalle wurden in concentrirte Lösung gelegt, diese zum Kochen erhitzt und $2-3$ Minuten

Fig. 7. darin erhalten. Auf der Basis entstehen dadurch rhombische a Grübchen, deren Längsrichtung der $b$-Axe parallel läuft, und auf dem Prisma monosymmetrische Figuren (Fig. 7a, b), mit

b

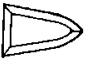

c der Längs- und Symmetrieaxe der Kante $\{001\}\{110\}$ parallel.

Nach der Behandlung mit kochender concentrirter Schwefelsäure (circa 4 Minuten) zeigen sich auf der Basis dieselben rhombenförmigen Vertiefungen, wie bei der Behandlung mit Aetznatron, dagegen treten auf dem Prisma diagonal gestellte, mehr oder minder unregelmässige viereckige Grubchen auf. Auf dem Makrodoma

Fig. 8.

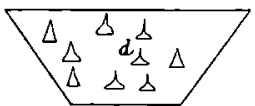

(102\} wurden im Allgemeinen Formen von dreieckigem bis fünfeckigem Umriss (Fig. 8) beobachtet, nur ein einziges Mal entstand eine einseitige Riefung parallel der Combinationskante (102): (1T0).

Wie zu erwarten war, wurden mit kohlensaurem Alkali und mit Aetznatron analoge Aetzfiguren erzielt, während die Schwefelsäure auf den Prismenflächen in anderer Weise einwirkte. Auffallend ist, dass auf $\{001\}$ die mit Schwefelsäure und die mit Aetznatron

*) Vergl. Ts chermak, Lehrb. d. Min. 2. Aufl. S. 140 . 
erhaltenen Figuren ubereinstimmen, wăhrend die mit kohlensaurem Alkali erhaltenen abweichen.

Die Beziehungen zwischen den natürlichen und den küstlichen Aetzfiguren sind einfach. Auf der Basis zeigen die mit Aetzoatron und mit Schwefelsăure, sowie die von T sch e rmak abgebildeten, mit kohlensaurem Natron erzeugten Eindrucke denselben rhombischen Umriss, wie die naturlichen Aetzfiguren von $\{001\}$, z. B. an dem Baryt vom Giftherge. Leider waren die von mir hergestellten kunstlichen Aelzfiguren zu klein, um eine Messung ihrer Flächen zuzulassen, so dass der Vergleich mit der krystallographisch bestimmten Fläche am Giftberger Vorkommen nicht specieller durchgefuhrt werden kann.

Auf den Prismenflächen ist die Aehnlichkeit der kunstlichen mit Aetznatron oder kohlensaurem Alkali erhaltenen (Fig. $7 \mathrm{a}$ und $7 \mathrm{~b}$ ) und der naturlichen von Gonnard abgebildeten (Fig. 7c) Aetzeindrucke eine auffallende. Die Unterschiede zwischen den künstlichen einerseits und manchen natürlichen andererseits sind nicht grösser, als die Verschiedenheilen der letzleren unter einander.

Auf die Gleichheit der kunstlichen und nalurlichen Aelzfiguren auf $\{102\}$ wurde schon oben hingewiesen. Es sind beide vorzugsweise von dreieckiger Gestalt.

Die Anordnung sowohl der natürlichen, als auch der künstlichen, monooder disymmetrischen Aetzfiguren entspricht in den mir bekannt gewordenen Fällen der Symmetrie der rhombischen Holoëdrie.

Ausnahmen machen nur in manchen Fällen die Basisfiguren des Kronthaler Vorkommens. Doch erschwerte hier die ausserordenlliche Zartheit und Kleinheit der Figuren die Untersuchung und verbietel es, auf diese Erscheinung hin für den Baryt Symmetrieverhältnisse anzunehmen, welche von denen der holoëdrisch-rhombischen Krystalle abweichen.

Scheinbar hemimorpher Krystalle des Baryts wird in der Literatur mehrfach Erwähnung*) getban. Von de Kalb hat Cbester ${ }^{* *}$ ) Krystalle beschriehen, die nach der Brachyaxe hemimorph ausgebildet erscheinen; Hankel***) erwähnt, dass er Krystallformen am Baryt gefunden habe, denen keine Symmetrie zukäme. Symmetrielos erscheint auch der oben erwähnte Krystall vom Kronthal (Fig. 2) durch seine asymmetrische Streifung.

Durch seine umfangreichen Untersuchungen uber das thermoëlektrische Verhalten des Baryls gelangt Hankel zu dem Resultate, dass demselben keine Hemimorphie eigen ist, dass die abnormen thermoëlektrischen

*) Rèus s, Sitzungsber. d. Wien. Akad. 1869, 59, Aprilheft.

**) Diese Zeitschr. 14, 297.

***) H an k e I, Abh. d. malh.-phys. Cl. d. k. sächs. Ges. d. Wiss. 10, 280. 
Erscheinungen, sowie die abnorme geometrische Gestalt, die oft der Symmetrie der holoeddrischen Ausbildung des Baryts nicht entspricht, lediglich durch Wachsthumsbedingungungen hervorgerufen werden. Offenbar kommt der Krystallgestalt und dem thermoëlektrischen Verhalten, welches letztere nach Hankel von dem Feblen oder Vorhandensein von Krystallfächen, also von äusseren Verhältnissen abhängen kann, eine geringere Bedeutung zu bei der Entscheidung der Frage, ob gewissen Krystallen eine hemimorphe Ausbildung eigen ist oder nicht, als den Aetzfiguren. Dies ist der Grund, weshalb bei der Untersuchung der Kronthaler Barytkrystalle, welche gewisse Anomalien in ihrer geometriscben Ausbildung zeigen, etwas näher auf die Gestalt und die Anordnung der kunstlichen wie der naturlichen Aetzfiguren eingegangen wurde. 